\title{
A musicoterapia como tratamento não-farmacológico para o Transtorno do Espectro Autista (TEA) infantil: uma revisão da literatura
}

\author{
Musicotherapy as a non-pharmacological treatment for childhood Autism Spectrum \\ Disorder (ASD): a literature review
}

La musicoterapia como tratamiento no farmacológico para el Desorden del Espectro Autista (DEA) infantil: revisión de la literatura

Rayssa Almeida Nogueira ${ }^{*}$, Miriana Figueiredo Pereira Paiva ${ }^{1}$, Lays Ferreira Fava ${ }^{1}$, Patrícia Anacleto Pires ${ }^{1}$, Jordana Rodrigues Jacinto ${ }^{1}$, Gabriela Santana Pimentel ${ }^{1}$, Rozileia Silva Leonardo'.

\section{RESUMO}

Objetivo: Dissertar sobre a atuação da musicoterapia na socialização de crianças acometidas pelo Transtorno do Espectro Autista (TEA), bem como os seus ef eitos no tratamento e a melhora na qualidade de vida. Revisão bibliográfica: A musicoterapia tem sido utilizada como uma forma de auxílio no tratamento do TEA. Estudos comprovam que a música é capaz de causar grande impacto nas áreas corticais do cérebro, gerando benefícios e influências positivas nas particularidades dos indivíduos. Além disso, as literaturas ressaltam a importância da musicoterapia no processo de socialização das crianças com TEA, evidenciando melhorias significativas no desenvolvimento de suas atividades diárias, percepções, controle, audição e ritualismos, o que possibilita a interação e comunicação compartilhada. Considerações finais: Pode-se perceber a eficácia e a abrangência da utilização da musicoterapia no tratamento do TEA, visto que essa terapia possibilita grandes impactos na qualidade de vida dos pacientes acometidos. Além disso, é poss ível constatar que $o$ ambiente e as relações interpessoais influenciam, de modo positivo ou negativo, na ef etividade do tratamento.

Palavras-chave: Autismo, Criança, Musicoterapia, Tratamento.

\begin{abstract}
Objective: To discuss the role of music therapy in the socialization of children affected by the Autistic Spectrum Disorder (ASD), as well as its effects on the treatment and improvement in quality of life. Bibliographic review: Music therapy has been used as an aid in the treatment of ASD. Studies prove that music can have a great impact on the cortical areas of the brain, generating benefits and positive influences on the particularities of individuals. Moreover, the literature highlights the importance of music therapy in the socialization process of children with ASD, showing significant improvements in the development of their daily activities, perceptions, control, listening, and ritualisms, which enables interaction and shared communication. Final considerations: We can perceive the efficacy and comprehensiveness of the use of music therapy in the treatment of ASD, since this therapy allows for great impacts on the quality of life of affected patients. Moreover, it is possib le to notice that the environment and interpersonal relationships influence, in a positive or negative way, the effectiveness of the treatment.
\end{abstract}

Keywords: Autism, Child, Musicotherapy, Treatment.

${ }^{1}$ Centro Universitário Redentor, Itaperuna - RJ. *E-mail: almeidarayssa008@gmail.com

SUBMETIDO EM: 12/2021

ACEITO EM: 12/2021

PUBLICADO EM: 12/2021

REAC | Vol. 39 | DOI: https://doi.org/10.25248/REAC .e9565.2021 


\section{RESUMEN}

Objetivo: Discutir el papel de la musicoterapia en la socialización de los niños af ectados por el Trastorno del Espectro Autista (TEA), así como sus efectos en el tratamiento y la mejora de la calidad de vida. Revisión bibliográfica: La musicoterapia se ha utilizad o como forma de ayuda en el tratamiento del TEA. Los estudios demuestran que la música es capaz de causar un gran impacto en las áreas corticales del cerebro, generando beneficios e influencias positivas en las particularidades de los individuos. Además, la literatura destaca la importancia de la musicoterapia en el proceso de socialización de los niños con TEA, mostrando mejoras significativas en el desarrollo de sus actividades cotidianas, percepciones, control, escucha y ritualismos, lo que permite la interacción y la comunicación compartida. Consideraciones finales: Se puede percibir la eficacia y amplitud del uso de la musicoterapia en el tratamiento del TEA, ya que esta terapia permite grandes impactos en la calidad de vida de los pacientes af ectados. Además, es posible percibir que el ambiente y las relaciones interpersonales influyen, de forma positiva o negativa, en la eficacia del tratamiento.

Palabras clave: Autismo, Musicoterapia, Niño, Tratamiento.

\section{INTRODUÇÃO}

O Transtorno do Espectro Autista (TEA) é um distúrbio pertencente ao grupo dos Transtornos Globais do Desenvolvimento (TGD), originário durante o neurodesenvolvimento e que se manifesta a partir do período da infância, sendo diagnosticado, em grande parte dos casos, na iniciação da vida escolar. Tem como característica a dificuldade na comunicação e interação social, possuindo padrões comportamentais repetitivos, em alguns casos desenvolvimento intelectual irregular, tendendo a levar o indivíduo autista ao auto isolamento, além de prejudicar os afazeres diários do acometido pelo transtorno (AMERICAN PSYCHIATRIC ASSOCIATION (APA), 2014; ONZI FZ e GOMES RF, 2015).

O TEA, embora apresente multifatores etiológicos, tem sido considerado o único transtorno de ordem neuropsiquiátrica que sofre influência direta da hereditariedade, ou seja, possui maior tendência a ser transmitido por meio da herança genética. Dessa forma, pais de uma criança acometida por TEA têm de $50 \%$ a 100\% de risco de terem um próximo filho com o mesmo transtorno (MECCA TP, et al., 2011).

Ademais, por ser um transtorno dividido em espectros, cada grau possui características particulares que requerem uma intervenção em âmbitos específicos. Diante disso, existem diferentes níveis de gravidade para o TEA, sendo que no primeiro, mais leve, o indivíduo requer apoio; no segundo, moderado, requer apoio substancial; e no terceiro, por sua vez, muito apoio substancial (APA, 2014). Contudo, é válido ressaltar, também, que as formas de intervenção não trazem a inexistência do transtorno, visto que o TEA permanece com o indivíduo durante todo o seu ciclo vital, mas auxiliam, indiscutivelmente, no processo de socialização e melhora comportamental dos autistas (ONZI FZ e GOMES RF, 2015).

Dentre alguns tratamentos utilizados, presentemente, com ênfase na melhora do quadro clínico dos pacientes acometidos pelo TEA, tem-se a musicoterapia, que vem trazendo significativos resultados (BRANDALISE A, 2013). A terapia envolvendo atividades musicais, utilizada pela medicina desde meados do século XX, vem sendo aplicada em diversos âmbitos do cuidado integral em saúde, visando melhorar o quadro dos pacientes (PADILHA MCP, 2008).

Em crianças portadoras do TEA, esse tratamento tem papel fundamental na indução ao aumento da responsividade clínica dos indivíduos, mediante a melhora na comunicação, interação, socialização, padrão comportamental e desenvolvimento intelectual da criança (FREIRE MH, et al., 2014). Além disso, essa modalidade de terapia busca o aprimoramento ou restauração de funções ind ividuais que possibilitem uma ampliação na qualidade de vida dos acometidos pelo transtorno e, desse modo, traz um maior potencial em torno dos resultados que são esperados ao finalizar o tratamento (SAKAI FA, et al., 2004).

O presente artigo teve como objetivo avaliar a utilização da musicoterapia e seus ef eitos comprovados no tratamento de crianças acometidas pelo Transtorno do Espectro Autista, apresentando, sequencialmente, a 
atuação da música como agente de intervenção direta no quadro clínico dos pacientes, seus ef eitos visíveis na comunicação e sociabilidade dos indivíduos, sua interferência no processo de desenvolvimento da capacidade intelectual dos acometidos pelo TEA e, sobretudo, como esse tratamento propicia o aumento da qualidade de vida das crianças, além de refletir positivamente em seus núcleos familiares.

\section{REVISÃO BIBLIOGRÁFICA}

\section{Efeitos corticais da música}

A música é capaz de construir um contato interpessoal, fazendo com que o acesso ao campo mental ocorra. Essa ponte, portanto, é realizada por meio da conexão feita pelas fibras nervosas entre o tálamo e hipotálamo com o cérebro, o que favorece a estimulação simultânea dessas áreas. Sendo assim, a música ganha destaque no âmbito da geração de prazer, uma vez que ao ocorrerem esses estímulos, as sensações de tensão emocional serão aliviadas (PIAZZETTA CMF, 2014).

Dessa forma, o cérebro pode ser dividido em três estruturas: cérebro cortical, cérebro básico e cérebro emocional. Dessa forma, o cérebro básico é responsável por regular funções fisiológicas e vegetativas e está altamente associado ao ritmo, quando se refere à música. O cérebro emocional, por sua vez, é responsável pela regulação das emoções, sendo formado pelo sistema límbico e corpo caloso. A melodia, portanto, é associada a essa estrutura. Já o cérebro cortical, é responsável pela percepção do presente, passado efuturo, sendo associado a harmonia musical. Sendo assim, o córtex com um todo é ativado, haja vista a extensa carga de energia provocada pelo estímulo sonoro (VARGAS MER, 2012).

Ademais, é possível inferir que devido ao ef eito causado pelo estímulo sonoro, ocorre a ativação do sistema recompensa do cérebro, liberando neurotransmissores como dopamina e serotonina (LINHARES L, et al., 2010). Esse fato, garante uma melhora no humor e uma maior aptidão a atividades que geram prazer. Contudo, o ef eito gerado pela excitação das áreas corticais, pode provocar percepções distintas confome o tipo de música, frequência ou melodia (AREIAS JC, 2016). Somado a esse fato, a música também atua diretamente no sistema nervoso autônomo apresentando como resposta, efeitos benéficos como alívio da dor. Nesse contexto, a teoria do portal medular da dor explica a relação sonora com esse evento, haja vista o fato da música agir de forma competitiva com a dor, modulando esse estímulo doloroso (TODRES, 2006).

Sendo assim, segundo Fernandes PRS (2012), as dimensões musicais trabalhadas podem englobar os setores físicos, mentais e psicológicos, sociais, bem como espirituais. No entanto, o ef eito sonoro em cada área apresenta consequências distintas, como por exemplo o relaxamento muscular presenciado no âmbito físico e o reforço da ideia de pertencimento nas dimensões psicológicas. Todavia, no âmbito espiritual, a música tem a capacidade terapêutica de torna-se "ponte" entre o meio físico e mental. Sendo assim, pode-se inferir a influência positiva da música na qualidade de vida de uma pessoa em contexto integral e amplo de saúde (físico, mental e espiritual).

\section{A musicoterapia no processo de socialização}

A música está atrelada à função de elevar os domínios cognitivos, emocionais, sociais, espirituais e terapêuticos, nos âmbitos do consciente e inconsciente. Essa atuação se estabelece por meio da união de elementos musicais, como a harmonia, melodia e o ritmo. No entanto, apesar dessa tríade trabalhar em conjunto na mente humana, há uma especificação no que tange aos aspectos motores (ritmo), af etivos (melodia) e intelectuais (harmonia). Sendo assim, constata-se que é por meio da música que o indivíduo constrói as suas primeiras noções de pertencimento a um determinado grupo cultural, bem como a noção de existência, principalmente, no meio social (FERNANDES PRS, 2012).

Nesse contexto, a perturbação do espectro do autismo (PEA) af eta diretamente a comunicação, integração social e capacidade de realizar jogos simbólicos. Diante disso, esses três conceitos se definem como tríade responsável por um padrão de comportamento restrito e repetitivo (PADILHA MCP, 2008). Dessa forma, crianças com PEA apresentam maior grau de dificuldade na comunicação verbal e não-verbal. Mediante a isso, como estratégia de comunicação, elas utilizam-se de recursos pré-simbólicos, como gritos, movimentos 
bruscos e manipulações, visando a possibilidade de intenções comunicativas. No entanto, os níveis de compreensão da informação e a forma com que são transmitidos não dialogam entre si, comprometendo, portanto, a interação social em um contexto geral (REIS HIS, et al., 2016).

Nesse viés, a musicoterapia apresenta um papel importante no tratamento e na melhoria da qualidade de vida de crianças autistas, principalmente no âmbito social. Dessa maneira, segundo Aires Filho SAA (2019), "a música é uma linguagem que acessa partes distintas do nosso cérebro, estimulando sinapses que beneficiam a interação humana, o processamento da fala e a comunicação de maneira geral". Nesse contexto, o campo da neurociência apresenta diversos estudos acerca desse tema, demonstrando a correlação direta e implícita dos sons com as emoções, uma vez que ocorre a presença de respostas fisiológicas positivas ou negativas, mediante estímulos sonoros externos (SAMPAIO RT, et al., 2015).

Além disso, a música funciona como promotora da ativação dos neurônios espelhos em crianças com PEA. Esses neurônios, por sua vez, são viso-motores que disparam tanto quando se realiza determinado ato como observar outro ser realizando o mesmo ato executado (PASSOS-FERREIRA C, 2011). Esse fato, corrobora para uma cascata de processos cognitivos e emocionais, que proporcionam a flexibilidade mental, o fortalecimento de vínculos, o compartilhamento de emoções, bem como o desenvolvimento da empatia. Sugerindo, portanto, a melhora na comunicação e interação social da criança com PEA (PEGORARO LC, 2017).

Segundo Wan C e Schlaug G (2010), os indivíduos acometidos pelo TEA possuem diferenças na massa total do cérebro, além de apresentarem diferenças de integração e simetria na conexão das regiões auditivas e motora e nas áreas de fala. Nessa mesma esteira, demais autores ratificam que os circuitos neurais associados ao processamento da fala e das canções (sons) são os mesmos, sendo que em indivíduos com TEA o processamento das canções é mais efetivamente ativado do que o processamento da fala propriamente dita (LAI G, et al., 2012; FABRICIUS T, 2012). Esses fatores, justificariam a utilização da musicoterapia no tratamento para TEA como forma de desenvolvimento da linguagem e controle das emoções.

Ademais, no que tange ao pensamento de Sampaio RT, et al. (2015), um dos objetivos da musicoterapia no tratamento de pessoas com TEA é conseguir ultrapassar o ritualismo na manipulação de objetos, comportamento típico em pessoas com TEA, para assim se desenvolver a atenção compartilhada, o que contribuiria no processo de interação e comunicação sociais. Nesse sentido, para Berger DS (2002), a musicoterapia deve ser aplicada através da mescla entre pulsações musicais regulares e previsíveis, o qual cria um ambiente não ameaçador à pessoa com TEA; e outros elementos musicais diferentes, os quais favorecem a flexibilidade e variação, fazendo com que haja melhora nos comportamentos estereotipados, invariáveis e inflexíveis presentes nos acometidos pelo TEA.

\section{A musicoterapia na melhoria da qualidade de vida}

Evidencia-se, apesar das bases fisiológicas da musicoterapia não serem bem conhecidas ainda, que a música atua na melhoria da qualidade de vida, influenciando em algumas variáveis da saúde, como as cerebrais e psicossociais. Sendo assim, a música possui caráter transformador tanto físico quanto psíquico, sendo uma forma de expressão de emoções e sentimentos abrangendo os campos sociais, ambientais, econômicos ou religiosos (AREIAS JC, 2016).

Nesse contexto, há uma correlação da música com a neuroplasticidade, uma vez que a prática musical intensiva levaria ao crescimento de áreas cerebrais como: frontal, motora, temporal e o corpo caloso, além de aumentar a conexão entre os lobos frontal e temporal em ambos os hemisférios, ativando áreas associadas a emoção (WAN C e SCHLAUG G, 2010). Nesse mesmo viés, segundo Fleury EAB e Santos KD (2016), observa-se que os portadores de TEA submetidos a musicoterapia passaram a apresentar maiores demonstrações não verbais de bem-estar e af eto como: o sorriso social, o prazer e satisfação com as sessões e a retribuição de gestos provenientes de outrem, entre eles o beijo e o abraço. Dessa forma, houve melhoria na qualidade de vida dos indivíduos com TEA, visto que sem a utilização da música os mesmos apresentam diferenças na massa total do cérebro e dificuldades para se controlar as emoções (WAN C e SCHLAUG G, 2010; FABRICIUS T, 2012). 
Nesse sentido, a música contribui para que os portadores de TEA ampliem suas capacidades físicas e motoras, fazendo com que haja desenvolvimento da sua percepção, sua audição e seu controle moto $r$. Sendo assim, com as atividades musicais ocorre o aprimoramento motor, visto que se verifica a movimentação com agilidade, o desenvolvimento de ritmo e o melhor controle dos músculos (MONTEIRO DHM e FERMOSELI AFO, 2014). Esses fatores propiciam o melhor equilíbrio e coordenação ao andar ou se movimentar, favorecendo também o processo do desenvolvimento da leitura e escrita (ARAÚJO NA, et al., 2018; GARCIA VP e SANTOS R, 2012).

Ademais, no âmbito educacional a musicoterapia provoca mudanças comportamentais nas crianças com TEA, fazendo com que haja melhor adaptação à vida escolar (ARAÚJO NA, et al., 2018). Nesse sentido, a música é utilizada como método de aprendizado, visto que através dela essas crianças conseguem interagir e se comunicar, melhorando o seu desempenho escolar. Sendo assim, as crianças com TEA que tinham acompanhamento com a musicoterapia e com atividades extramusicais, apresentavam melhorias na concentração, no desenvolvimento da af etividade, na expressão dos sentimentos, no desenvolvimentos das funções cognitivas e na criatividade (BARBOSA JIC e BORBA A, 2010; MONTEIRO DHM e FERMOSELI AFO, 2014).

Outrossim, o tratamento através da musicoterapia traz melhorias para o relacionamento familiar. Segundo Benenzon R (1987), a musicoterapia pode promover melhorias na saúde da família por meio da comunicação não verbal. Barbosa AJG e Oliveira LD (2008) afirmam que nessas famílias, principalmente quando um dos familiares se abdica da vida profissional para os cuidados do filho, podem ocorrero aparecimento de sintomas depressivos, sentimentos de desvalia e estresse parental. Sendo assim, a musicoterapia se coloca como uma terapia de apoio e suporte familiar, visto que gera benefícios para a relação entre pais e filhos, sobretudo o principal cuidador do indivíduo com TEA, e gera diminuição nos sintomas supracitados de acordo com que há evolução no tratamento do indivíduo portador do TEA (BARBOSA AD, et al., 2018; FREIRE MH, et al., 2014).

Acredita-se que a musicoterapia beneficia no tratamento das doenças e transtornos, haja vista que a música está empregada no uso de várias modalidades ao se depararem com um discente estudando e ouvindo música, por exemplo, e, assim, é perceptível o êxito em aliviar a tensão e o estresse acometido pela maioria dos alunos. Dito isso, os apaixonados pela música vivenciam como essas sensações de prazer das sinfonias implicam em uma terapia relaxante e muitas das vezes criativa. Logo, isso não seria diferente com pacientes diagnosticados com o Transtorno do Espectro Autista (TEA), pois a possibilidade que eles têm de se comunicar e aprender culturalmente e socialmente algo de forma divertida é notável. Alguns estudos relatam que a música está sendo cada vez mais empregada em pacientes com enfermidades, visto que o som ajuda a relaxar e prevalece no tratamento e recuperação de doentes (SAKAI FA, et al., 2004; PADILHA MCP, 2008; MONTEIRO DHM e FERMOSELI AFO, 2014).

Outrossim, resultados mostram como a musicoterapia atua no cérebro desses pacientes ao ativar as regiões do Hipotálamo, Tálamo e Hipocampo onde, respectivamente, atua na regulação da temperatu ra e o prazer de comer, além de ativar os sentidos e o centro da memória estimulando o aprendizad o (BARBOSA JIC e BORBA A, 2010; AREIAS JC, 2016). Ademais, Freire MH, et al. (2014) ressalta que o uso da musicoterapia está definitivamente sendo empregada com sucesso em diferentes tipos de espectros e distintas idades, desde as crianças mais novas até as mais avançadas.

Contudo, isso discute-se a partir de Landa RJ (2008) no momento que uma parcela estaria relacionada a uma melhoria conforme a idade, onde pressupõe em tal embasamento que quanto mais novo o portador com TEA, mais ele apresenta aprendizado. Uma vez que as crianças em torno da $2^{\underline{a}}$ idade são classificadas em "Autonomia versus Dúvida e Vergonha" segundo o Complexo de Édipo, onde a criança dos 18 meses aos 3 anos está apta a explorar o seu meio ambiente que está em volta dela favorecendo o uso da musicoterapia. Além disso, a $3^{a}$ idade classifica em "Iniciativa versus culpa" em que o Complexo de Édipo entra em ascendência à medida que a criança toma iniciativa desenvolvendo a curiosidade das suas próprias atividades, e quando não concluída, se culpabiliza. 


\section{CONSIDERAÇÕES FINAIS}

Sendo assim, é importante ressaltar que a musicoterapia como tratamento não -farmacológico para o TEA infantil apresenta grande eficácia e consideráveis evoluções benéficas em diferentes quadros clínicos, tendo em vista seus impactos na qualidade de vida dos pacientes acometidos ao atuar nos setores físicos, psicológicos, sociais e espirituais. Ademais, pôde-se constatar melhorias exponenciais no processo de socialização de crianças com TEA, haja vista a estimulação de sinapses no campo da interação humana, englobando fala e comunicação. Observou-se, também, melhorias no âmbito educacional, bem como na adaptação à vida escolar. Desse modo, infere-se que o tratamento com musicoterapia, aliado a um ambiente com interações interpessoais positivas, gera uma melhor qualidade de vida em pacientes infantis com TEA.

\section{REFERÊNCIAS}

1. AIRES FILHO SAA. Música é linguagem? E o que o autismo tem a ver com isso?. XXIX Congresso da Anppom. 2019: $1-8$.

2. AMERICAN PSYCHIATRIC ASSOCIATION (APA). Manual diagnóstico e estatístico de transtornos mentais. 5. ed. Artmed, 2014. Disponível em: http:/www.niip.com.br/wp-content/uploads/2018/06/Manual-Diagnosico-e-Estatisticode-Transtornos-Mentais-DSM-5-1-pdf. Acessado em:20 de dezembro de 2021.

3. ARAÚJO NA, et al. A musicoterapia no tratamento de crianças com autismo: revisão integrativa. ReonFacema, 2018; 4(2): 1102-1106.

4. AREIAS JC. A música, a saúde e o bem-estar. Nascer e Crescer, 2016;25(1):7-10.

5. BARBOSA AD, et al. $A$ não adesão de pais de crianças com Transtorno do Espectro do Autismo em grupo de musicoterapia:um relato de experiência. Revista de Musicoterapia, 2018;24:26-44.

6. BARBOSA AJG, OLIVEIRA, LD. Estresse e enfrentamento em pais de pessoas com necessidades especiais. Psicologia em Pesquisa, 2008;2(2): 36-50.

7. BARBOSA JIC, BORBA A. O surgimento das terapias cognitivo-comportamentais e suas consequências para 0 desenvolvimento de uma abordagem clínica analítico-comportamental dos eventos privados. Revista Brasileira de Terapia Comportamental, 2010;12:1-2.

8. BENENZON R. O autismo, a família, a instituição e a Musicoterapia. Enelivros, 1987;364p.

9. BERGER DS. Music Therapy, Sensory Integration and the Autistic Child. Jessica Kingsley Jessica Publishers, 2002; $255 \mathrm{p}$.

10. BRANDALISE A. Musicoterapia aplicada à pessoa com transtorno do espectro do autismo (TEA): uma revisão sistemática. Rev Bras Musicoterapia, 2013;15(15): 28-42.

11. FABRICIUS T. On neural systems for speech and song in autism. Brain, Oxford University Press (OUP), 2012; 135(11).

12. FERNANDES PRS. Sons e silêncios: musicoterapia no tratamento de indivíduos com perturbações do espectro do autismo. Dissertação (Mestrado em Ciências da Educação): Universidade Católica Portuguesa, 2012;128 p.

13. FLEURY EAB, SANTOS KD. Musicoterapia na interação social de pessoas com TEA: estudo de revisão. Revista Incantare, 2016; 7(2): 1-19.

14. FREIRE MH, et al. Efeitos da Musicoterapia Improvisacional no tratamento de crianças com Transtorno do Espectro do Autismo. Dissertação (Mestrado em Neurociências - Neuropsiquiatria Clínica): Universidade Federal de Minas Gerais, 2014.

15. GARCIA VP, SANTOS R. A importância da utilização da música na educação infantil. Revista Digital, 2012;00(169): 1-10.

16. LAI G, et al. Neural systems for speech and song in autism. Brain, Oxford University Press (OUP), 2012; 135(3):961975.

17. LANDA RJ. Diagnosis of autism spectrum disorders in the first 3 years of life. Nature Clinical Practice Neurology, 2008;4(3): 138-47.

18. LINHARES L, et al. A música como recurso para o desenvolvimento do projeto de humanização realizado no Hospital Municipal Odilon Behrens. Formação@ Docente, 2010;2(1): 44-50.

19. MECCA TP, et al. Rastreamento de sinais e sintomas de transtornos do espectro do autismo em irmãos. Revista de Psiquiatria do Rio Grande do Sul, 2011;33(2): 116-120.

20. MONTEIRO DHM, FERMOSELI AFO. Musicoterapia: contribuição como ferramenta terapêutica no auxílio a tratamento de patologias adversas inseridas no âmbito da saúde. Ciências Biológicas e da Saúde, 2014;2(2):91-110.

21. ONZI FZ, GOMES RF. Transtorno do Espectro Autista: a importância do diagnóstico e reabilitação. Cademo Pedagógico, 2015;12(3):188-199. 
22. PADILHA MCP. A musicoterapia no tratamento de crianças com perturbação do espectro do autismo. Dissertação (Mestrado em Medicina):Universidade da Beira Interior. 2008.

23. PASSOS-FERREIRA C. Seria a moralidade determinada pelo cérebro? Neurônios-espelhos, empatia e neuromoralidade. Physis: Revista de Saúde Coletiva, 2011;21:471-490.

24. PEGORARO LC. A música como intervenção neuropsicológica no tratamento do transtorno do espectro autista (TEA): uma revisão crítica da literatura. Trabalho de Conclusão de Curso (Especialização em Psicologia - Ênfase em Neuropsicologia) - Instituto de Psicologia. Universidade Federal do Rio Grande do Sul, Porto Alegre, 2017;27p.

25. PIAZZETTA CMF. Música em Musicoterapia: estudos e reflexões na construção do corpo teórico da Musicoterapia. Revista InCantare, 2014;1:38-69.

26. REIS HIS, et al. Características e especificidades da comunicação social na perturbação do espectro do autismo. Revista Brasileira de Educação Especial, 2016;22(3):325-336.

27. SAKAI FA, et al. Musicoterapia corporal. Convenção Brasil Latino América, Congresso Brasileiro e Encontro Paranaense de Psicoterapias Corporais, 2004;9p.

28. SAMPAIO RT, et al. A Musicoterapia e o Transtorno do Espectro do Autismo: uma abordagem informada pelas neurociências para a prática clínica. Per musi, 2015;32: 137-170.

29. VARGAS MER. Influências da música no comportamento humano: explicações da neurociência e psicologia. Anais do Congresso Internacional da Faculdade EST, 2012; 944-956.

30. WAN C, SCHLAUG G. Neural pathways for language in autism: the potential for music based treatments. Future Neurol., 2010; 5(6): 797-805. 